\title{
Harmonic numbers of order two
}

\author{
Anthony Sofo
}




\title{
HARMONIC NUMBERS OF ORDER TWO
}

\section{ANTHONY SOFO}

Received 21 October, 2011

\begin{abstract}
We investigate sums of Euler type, in particular the summation, in closed form, of the
\end{abstract} product of Harmonic numbers of order two and the square of reciprocal binomial coefficients.

2000 Mathematics Subject Classification: 05A10; 11B65; 11M06; 33B15; 33D60; 33C20

Keywords: harmonic numbers, Riemann Zeta functions, binomial coefficients, series representations

\section{INTRODUCTION}

There are various identities in the literature for harmonic number sums in higher powers. A result that goes back to the time of Euler, see [17], or [19] is

$$
\sum_{n=1}^{\infty} \frac{H_{n}^{(p)}}{n^{p}}=\frac{1}{2}\left(\zeta^{2}(p)+\zeta(2 p)\right)
$$

where $\zeta(z)=\sum_{r=1}^{\infty} \frac{1}{r^{z}}, \mathbb{R}(z)>1$, denotes the familiar Riemann zeta function.

$$
H_{n}=\gamma+\psi(n+1)=\sum_{r=1}^{n} \frac{1}{r}=\int_{0}^{\infty} \frac{1-t^{n}}{1-t} d t
$$

is the $n^{t h}$ harmonic number and $\gamma$ denotes the Euler-Mascheroni constant. The generalized $n^{\text {th }}$ harmonic number in power $r, H_{n}^{(r)}$, is defined for positive integers $n$ and $r$ as

$$
H_{n}^{(r)}:=\sum_{m=1}^{n} \frac{1}{m^{r}}=\frac{(-1)^{r-1}}{(r-1) !}\left(\psi^{(r-1)}(n+1)-\psi^{(r-1)}(1)\right),
$$

where

$$
\psi^{(m)}(z)=(-1)^{m+1} m ! \sum_{r=0}^{\infty} \frac{1}{(z+r)^{m+1}}=(-1)^{m+1} m ! \zeta(m+1, z)
$$


are the polygamma functions of order $m$ which are defined by $\psi^{(0)}(z) \equiv \psi(z)$ and $\psi^{(m)}(z):=d^{m} \psi(z) / d z^{m}, m \in \mathbb{N}$ and $z \neq 0,-1,-2, \ldots$. Here $\psi(z)$ is the psi, or digamma, function, given as the logarithmic derivative of the well-known gamma function $\Gamma(z)$, i.e. $\psi(z):=d \log \Gamma(z) / d z . \zeta(\alpha, z)$ denotes the Hurwitz zeta function. There also exists the relation, which will be useful in our later analysis, between the polygamma functions and the generalized $n^{t h}$ harmonic number

$$
H_{n}^{(r+1)}=\zeta(r+1)+\frac{(-1)^{r}}{r !} \psi^{(r)}(n+1)
$$

and the recurrence relations

$$
\psi^{(m)}(z+1)=\frac{(-1)^{m} m !}{z^{m+1}}+\psi^{(m)}(z), H_{n+1}^{(r)}=\frac{1}{(n+1)^{p}}+H_{n}^{(r)} .
$$

In the paper [15] the author gave the result

$$
\sum_{n=1}^{\infty} \frac{H_{n}^{(2)}}{\left(\begin{array}{c}
n+k \\
k
\end{array}\right)}=\frac{k}{k-1}\left(\zeta(2)-H_{k-1}^{(2)}\right) .
$$

and in this paper we shall extend (1.3) by providing identities for the general sums

$$
\sum_{n=1}^{\infty} \frac{H_{n}^{(2)}}{n\left(\begin{array}{c}
n+k \\
k
\end{array}\right)^{2}}, \sum_{n=1}^{\infty} \frac{H_{n}^{(2)}}{\left(\begin{array}{c}
n+k \\
k
\end{array}\right)^{2}} \text { and } \sum_{n=1}^{\infty} \frac{H_{n}^{(2)}}{(n+1)\left(\begin{array}{c}
n+k \\
k
\end{array}\right)^{2}}
$$

A result that goes back to Euler [8] is listed in [3] or [9] and we shall refer to in the latter part of this paper is the following:

$$
\sum_{n=1}^{\infty} \frac{H_{n}^{(p)}}{n^{q}}=\zeta(p+q)+\sum_{n=1}^{\infty} \frac{H_{n}^{(p)}}{(n+1)^{q}}
$$

where

$$
\begin{gathered}
\sum_{n=1}^{\infty} \frac{H_{n}^{(p)}}{(n+1)^{q}}=\frac{A}{2}\left[\left(\begin{array}{c}
p+q \\
p
\end{array}\right)-A\right] \zeta(p+q)+B \zeta(p) \zeta(q) \\
-A \sum_{j=1}^{p+q}\left[\left(\begin{array}{c}
2 j-2 \\
p-1
\end{array}\right)+\left(\begin{array}{c}
2 j-2 \\
q-1
\end{array}\right)\right] \zeta(2 j-1) \zeta(p+q-2 j+1),
\end{gathered}
$$

here

$$
A=\left\{\begin{array}{c}
1, \text { for } p \text { odd, } q \text { even } \\
-1, \text { for } p \text { even, } q \text { odd }
\end{array}, \quad B=\left\{\begin{array}{l}
1, \text { for } p \text { odd, } q \text { even } \\
0, \text { for } p \text { even, } q \text { odd }
\end{array}\right.\right.
$$


Many finite versions of higher order harmonic number sum identities also exist in the literature, for example, see [11], [12], and [13]. Other finite sum identities can be seen in [18]. Further work in the summation of harmonic numbers and binomial coefficients has also been done by Sofo [16]. The works of, [1], [2], [4], [6], [5], [7], [10], [14], and [20] and references therein, also investigate various representations of binomial sums and zeta functions in simpler form by the use of the Beta function and other techniques. The following results will be useful for later analysis.

Lemma 1. Let $a>1$ be a positive integer then

$$
\sum_{n=1}^{\infty} \frac{H_{n}^{(2)}}{n(n+a)}=\frac{1}{a} \zeta(3)+\frac{1}{a} \zeta(2) H_{a-1}-\frac{1}{a} \sum_{j=1}^{a-1} \frac{H_{j}}{j^{2}}
$$

and

$$
\begin{aligned}
\sum_{n=1}^{\infty} \frac{H_{n}^{(2)}}{n(n+a)^{2}} & =-\frac{3}{4 a} \zeta(4)+\frac{1}{a^{2}} \zeta(3)+\frac{1}{a} \zeta(2)\left(\frac{H_{a-1}}{a}+2 H_{a-1}^{(2)}\right) \\
& -\frac{1}{a} \sum_{j=1}^{a-1}\left(\frac{H_{j}}{a j^{2}}+\frac{2 H_{j}}{j^{3}}+\frac{H_{j}^{(2)}}{j^{2}}\right)
\end{aligned}
$$

Proof. Consider

$$
\sum_{n=1}^{\infty} \frac{H_{n}^{(2)}}{n(n+a)}=\sum_{n=1}^{\infty} \sum_{k=1}^{n} \frac{1}{k^{2} n(n+a)}
$$

since these sums are absolutely convergent, by a re-arrangement of the double sum we can write

$$
\begin{aligned}
\sum_{n=1}^{\infty} \sum_{k=1}^{n} \frac{1}{k^{2} n(n+a)} & =\sum_{k=1}^{\infty} \sum_{n=0}^{\infty} \frac{1}{k^{2}(n+k)(n+a+k)} \\
& =\sum_{k=1}^{\infty} \frac{1}{a k^{2}}[\psi(a+k)-\psi(k)] .
\end{aligned}
$$

Now

$$
\sum_{k=1}^{\infty} \frac{1}{a k^{2}}[\psi(a+k)-\psi(k)]=\sum_{k=1}^{\infty} \frac{1}{a k^{3}}+\sum_{j=1}^{a-1} \sum_{k=1}^{\infty} \frac{1}{a k^{2}(k+j)}
$$




$$
=\frac{1}{a} \zeta(3)+\frac{1}{a} \sum_{j=1}^{a-1} \int_{0}^{1} x^{j-1} L i_{2}(x) d x
$$

where the Polylogarithmic function

$$
\operatorname{Li}_{2}(\beta)=\sum_{r \geq 1} \frac{\beta^{r}}{r^{2}} .
$$

By partial fraction decomposition we have

$$
\begin{gathered}
\sum_{j=1}^{a-1} \sum_{k=1}^{\infty} \frac{1}{a k^{2}(k+j)}=\sum_{j=1}^{a-1} \frac{1}{a} \sum_{k=1}^{\infty}\left[\frac{1}{j k^{2}}-\frac{1}{j k(k+j)}\right] \\
=\sum_{j=1}^{a-1} \frac{1}{a}\left[\frac{\zeta(2)}{j}-\frac{H_{j}}{j^{2}}\right]=\frac{H_{a-1} \zeta(2)}{a}-\frac{1}{a} \sum_{j=1}^{a-1} \frac{H_{j}}{j^{2}}
\end{gathered}
$$

and the result (1.6) follows. To prove (1.7) consider

$$
\sum_{n=1}^{\infty} \frac{H_{n}^{(2)}}{n(n+a)^{2}}=\sum_{n=1}^{\infty} \sum_{k=1}^{n} \frac{1}{k^{2} n(n+a)^{2}}
$$

since these sums are absolutely convergent, by a re-arrangement of the double sum we can write

$$
\begin{gathered}
\sum_{n=1}^{\infty} \sum_{k=1}^{n} \frac{1}{k^{2} n(n+a)^{2}}=\sum_{k=1}^{\infty} \sum_{n=0}^{\infty} \frac{1}{k^{2}(n+k)(n+a+k)^{2}} \\
=\sum_{k=1}^{\infty} \frac{1}{a^{2} k^{2}}\left[\psi(a+k)-\psi(k)-a \psi^{\prime}(a+k)\right] \\
=\sum_{k=1}^{\infty} \frac{1}{a^{2} k^{3}}+\sum_{j=1}^{a-1} \sum_{k=1}^{\infty} \frac{1}{a^{2} k^{2}(k+j)}+\sum_{j=1}^{a-1} \sum_{k=1}^{\infty} \frac{1}{a k^{2}(k+j)^{2}}-\sum_{k=1}^{\infty} \frac{\psi^{\prime}(1+k)}{a k^{2}} \\
=\frac{\zeta(3)}{a^{2}}+\sum_{j=1}^{a-1} \frac{1}{a^{2}}\left(\frac{\zeta(2)}{j}-\frac{H_{j}}{j^{2}}\right)+\frac{1}{a} \sum_{j=1}^{a-1} \sum_{k=1}^{\infty}\left(\begin{array}{c}
\frac{1}{k^{2} j^{2}}+\frac{1}{j^{2}(k+j)^{2}} \\
-\frac{2}{j^{2} k(k+j)}
\end{array}\right)
\end{gathered}
$$




$$
\begin{aligned}
& -\sum_{k=1}^{\infty} \frac{\zeta(2)-H_{k}^{(2)}}{a k^{2}} \\
& =\frac{\zeta(3)}{a^{2}}+\sum_{j=1}^{a-1} \frac{1}{a^{2}}\left(\frac{\zeta(2)}{j}-\frac{H_{j}}{j^{2}}\right) \\
& +\frac{1}{a} \sum_{j=1}^{a-1}\left(\frac{\zeta(2)}{j^{2}}-\frac{2 H_{j}}{j^{3}}+\frac{\zeta(2)}{j^{2}}-\frac{H_{j}^{(2)}}{j^{2}}\right)-\frac{\zeta^{2}(2)}{a} \\
& +\sum_{k=1}^{\infty} \frac{H_{k}^{(2)}}{a k^{2}} .
\end{aligned}
$$

From (1.1) we know that

$$
\sum_{k=1}^{\infty} \frac{H_{k}^{(2)}}{a k^{2}}=\frac{1}{2 a}\left(\zeta^{2}(2)+\zeta(4)\right)=\frac{7}{4 a} \zeta(4), \text { and also } \zeta^{2}(2)=\frac{5}{2} \zeta(4),
$$

hence

$$
\begin{aligned}
\sum_{n=1}^{\infty} \frac{H_{n}^{(2)}}{n(n+a)^{2}} & =-\frac{3}{4 a} \zeta(4)+\frac{1}{a^{2}} \zeta(3)+\frac{1}{a} \zeta(2)\left(\frac{H_{a-1}}{a}+2 H_{a-1}^{(2)}\right) \\
& -\frac{1}{a} \sum_{j=1}^{a-1}\left(\frac{H_{j}}{a j^{2}}+\frac{2 H_{j}}{j^{3}}+\frac{H_{j}^{(2)}}{j^{2}}\right) .
\end{aligned}
$$

Some special cases are noted in the remark.

Remark 1. When $a=0$

$$
\sum_{n=1}^{\infty} \frac{H_{n}^{(2)}}{n^{2}}=\frac{7}{4} \zeta(4), \sum_{n=1}^{\infty} \frac{H_{n}^{(2)}}{n^{3}}=3 \zeta(2) \zeta(3)-\frac{9}{2} \zeta(5),
$$

for $a=1$ and 5 and also using (1.3) and (1.4), we have, respectively

$$
\sum_{n=1}^{\infty} \frac{H_{n}^{(2)}}{n(n+1)}=\zeta(3), \sum_{n=1}^{\infty} \frac{H_{n}^{(2)}}{n(n+5)}=\frac{\zeta(3)}{5}+\frac{5 \zeta(2)}{12}-\frac{2953}{8640},
$$


and

$$
\begin{gathered}
\sum_{n=1}^{\infty} \frac{H_{n}^{(2)}}{n(n+1)^{2}}=\zeta(3)-\frac{3}{4} \zeta(4), \\
\sum_{n=1}^{\infty} \frac{H_{n}^{(2)}}{n(n+5)^{2}}=\frac{\zeta(3)}{25}-\frac{3 \zeta(4)}{20}+\frac{47 \zeta(2)}{72}-\frac{51499}{57600} .
\end{gathered}
$$

A related Lemma which will be useful later is the following.

Lemma 2. Let $a>1$ be a positive integer then

$$
\sum_{n=1}^{\infty} \frac{H_{n}^{(2)}}{(n+1)^{2}(n+a)}=\frac{3 \zeta(4)}{4(a-1)}-\frac{\zeta(2) H_{a-1}}{(a-1)^{2}}+\frac{1}{(a-1)^{2}} \sum_{j=1}^{a-1} \frac{H_{j}}{j^{2}}
$$

and

$$
\begin{aligned}
\sum_{n=1}^{\infty} \frac{H_{n}^{(2)}}{(n+1)^{2}(n+a)^{2}} & =\frac{3 \zeta(4)}{2(a-1)^{2}}-\frac{2 \zeta(2) H_{a-1}}{(a-1)^{3}}-\frac{2 \zeta(2) H_{a-1}^{(2)}}{(a-1)^{2}} \\
& +\frac{1}{(a-1)^{2}} \sum_{j=1}^{a-1}\left(\frac{2 H_{j}}{(a-1) j^{2}}+\frac{2 H_{j}}{j^{3}}+\frac{H_{j}^{(2)}}{j^{2}}\right)
\end{aligned}
$$

Proof. Consider

$$
\sum_{n=1}^{\infty} \frac{H_{n}^{(2)}}{(n+1)^{2}(n+a)}=\sum_{n=1}^{\infty} \sum_{k=1}^{n} \frac{1}{k^{2}(n+1)^{2}(n+a)}
$$

since these sums are absolutely convergent, by a re-arrangement of the double sum we can write

$$
\begin{aligned}
\sum_{n=1}^{\infty} \sum_{k=1}^{n} \frac{1}{k^{2}(n+1)^{2}(n+a)} & =\sum_{k=1}^{\infty} \sum_{n=0}^{\infty} \frac{1}{k^{2}(n+1+k)^{2}(n+a+k)} \\
& =\sum_{k=1}^{\infty} \frac{1}{k^{2}}\left[\frac{\psi(1+k)-\psi(a+k)}{(a-1)^{2}}+\frac{\psi^{\prime}(1+k)}{(a-1)}\right] .
\end{aligned}
$$

Now

$$
\sum_{k=1}^{\infty} \frac{1}{k^{2}}\left[\frac{\psi(1+k)-\psi(a+k)}{(a-1)^{2}}+\frac{\psi^{\prime}(1+k)}{(a-1)}\right]=-\sum_{j=1}^{a-1} \sum_{k=1}^{\infty} \frac{1}{(a-1)^{2} k^{2}(k+j)}
$$




$$
\begin{aligned}
& +\sum_{k=1}^{\infty} \frac{\zeta(2)-H_{k}^{(2)}}{(a-1) k^{2}} \\
= & \frac{\zeta^{2}(2)}{a-1}-\sum_{k=1}^{\infty} \frac{H_{k}^{(2)}}{(a-1) k^{2}}-\sum_{j=1}^{a-1} \sum_{k=1}^{\infty} \frac{1}{(a-1)^{2} k^{2}(k+j)} \\
= & \frac{\zeta^{2}(2)}{a-1}-\sum_{k=1}^{\infty} \frac{H_{k}^{(2)}}{(a-1) k^{2}}-\sum_{j=1}^{a-1} \frac{1}{(a-1)^{2}}\left(\frac{\zeta(2)}{j}-\frac{H_{j}}{j^{2}}\right) \\
= & \frac{\zeta^{2}(2)}{a-1}-\frac{1}{2(a-1)}\left(\zeta^{2}(2)+\zeta(4)\right)-\frac{\zeta(2) H_{a-1}}{(a-1)^{2}}+\sum_{j=1}^{a-1} \frac{H_{j}}{(a-1)^{2} j^{2}} \\
= & \frac{3 \zeta(4)}{4(a-1)}-\frac{\zeta(2) H_{a-1}}{(a-1)^{2}}+\frac{1}{(a-1)^{2}} \sum_{j=1}^{a-1} \frac{H_{j}}{j^{2}} .
\end{aligned}
$$

To prove (1.9) consider

$$
\begin{aligned}
& \sum_{n=1}^{\infty} \frac{H_{n}^{(2)}}{(n+1)^{2}(n+a)^{2}}=\sum_{n=1}^{\infty} \sum_{k=1}^{n} \frac{1}{k^{2}(n+1)^{2}(n+a)^{2}} \\
= & \sum_{k=1}^{\infty} \sum_{n=0}^{\infty} \frac{1}{k^{2}(n+1+k)^{2}(n+a+k)^{2}} \\
= & \sum_{k=1}^{\infty} \frac{1}{(a-1)^{3} k^{2}}\left[2 \psi(1+k)-2 \psi(a+k)+(a-1)\left(\psi^{\prime}(1+k)+\psi^{\prime}(a+k)\right)\right] \\
= & \sum_{k=1}^{\infty} \frac{1}{(a-1)^{3} k^{2}}\left[-2 \sum_{j=1}^{a-1} \frac{1}{(k+j)}+(a-1)\left(2 \psi^{\prime}(1+k)+\sum_{j=1}^{a-1} \frac{1}{(k+j)^{2}}\right)\right] \\
= & -\frac{2}{(a-1)^{3}} \sum_{j=1}^{a-1}\left(\frac{\zeta(2)}{j}-\frac{H_{j}}{j^{2}}\right)+\frac{2}{(a-1)^{2}} \sum_{k=1}^{\infty} \frac{\zeta(2)-H_{k}^{(2)}}{k^{2}} \\
- & \frac{1}{(a-1)^{2}} \sum_{j=1}^{a-1} \sum_{k=1}^{\infty} \frac{1}{(k+j)^{2}}
\end{aligned}
$$




$$
\begin{aligned}
& =\frac{3 \zeta(4)}{2(a-1)^{2}}-\frac{2 \zeta(2) H_{a-1}}{(a-1)^{3}}-\frac{2 \zeta(2) H_{a-1}^{(2)}}{(a-1)^{2}} \\
+ & \frac{1}{(a-1)^{2}} \sum_{j=1}^{a-1}\left(\frac{2 H_{j}}{(a-1) j^{2}}+\frac{2 H_{j}}{j^{3}}+\frac{H_{j}^{(2)}}{j^{2}}\right)
\end{aligned}
$$

and the result (1.9) is attained.

Remark 2. Some examples are

$$
\sum_{n=1}^{\infty} \frac{H_{n}^{(2)}}{(n+1)^{3}}=3 \zeta(2) \zeta(3)-\frac{11}{2} \zeta(5), \sum_{n=1}^{\infty} \frac{H_{n}^{(2)}}{(n+1)^{2}(n+2)}=\frac{3 \zeta(4)}{4}-\zeta(2)
$$

and

$$
\begin{aligned}
\sum_{n=1}^{\infty} \frac{H_{n}^{(2)}}{(n+1)^{4}} & =\zeta^{2}(3)+\frac{8}{3} \zeta(2) \zeta(4)-6 \zeta(6), \sum_{n=1}^{\infty} \frac{H_{n}^{(2)}}{(n+1)^{2}(n+5)^{2}}=\frac{3 \zeta(4)}{32} \\
& -\frac{35}{144} \zeta(2)+\frac{3827}{12288} .
\end{aligned}
$$

Lemma 3. Let $a>1$ be a positive integer then

$$
\begin{aligned}
\sum_{n=1}^{\infty} \frac{H_{n}^{(2)}}{(n+1)^{3}(n+a)} & =\frac{3 \zeta(2) \zeta(3)}{(a-1)}-\frac{11 \zeta(5)}{2(a-1)}-\frac{3 \zeta(4)}{4(a-1)^{2}} \\
& +\frac{\zeta(2) H_{a-1}}{(a-1)^{3}}-\frac{1}{(a-1)^{3}} \sum_{j=1}^{a-1} \frac{H_{j}}{j^{2}}
\end{aligned}
$$

and

$$
\begin{gathered}
\sum_{n=1}^{\infty} \frac{H_{n}^{(2)}}{(n+1)^{3}(n+a)^{2}}=\frac{3 \zeta(2) \zeta(3)}{(a-1)^{2}}-\frac{11 \zeta(5)}{2(a-1)^{2}}-\frac{9 \zeta(4)}{4(a-1)^{3}}+\frac{2 \zeta(2) H_{a-1}^{(2)}}{(a-1)^{3}} \\
+\frac{3 \zeta(2) H_{a-1}}{(a-1)^{4}}-\frac{1}{(a-1)^{3}} \sum_{j=1}^{a-1}\left(\frac{3 H_{j}}{(a-1) j^{2}}+\frac{2 H_{j}}{j^{3}}+\frac{H_{j}^{(2)}}{j^{2}}\right)
\end{gathered}
$$

Proof. Consider

$$
\sum_{n=1}^{\infty} \frac{H_{n}^{(2)}}{(n+1)^{3}(n+a)}=\sum_{n=1}^{\infty} \sum_{k=1}^{n} \frac{1}{k^{2}(n+1)^{3}(n+a)}
$$


since these sums are absolutely convergent, by a re-arrangement of the double sum we can write

$$
\begin{aligned}
& \sum_{n=1}^{\infty} \sum_{k=1}^{n} \frac{1}{k^{2}(n+1)^{3}(n+a)}=\sum_{k=1}^{\infty} \sum_{n=0}^{\infty} \frac{1}{k^{2}(n+1+k)^{3}(n+a+k)} \\
&=\frac{1}{2(a-1)^{3}} \\
& \sum_{k=1}^{\infty} \frac{1}{k^{2}}[2 \psi(a+k)-2 \psi(1+k)\left.-2(a-1) \psi^{\prime}(1+k)-(a-1)^{2} \psi^{\prime \prime}(1+k)\right] \\
&=\frac{1}{(a-1)^{3}} \\
& \sum_{j=1}^{a-1} \sum_{k=1}^{\infty} \frac{1}{k^{2}(k+j)}-\frac{1}{(a-1)^{2}} \sum_{k=1}^{\infty} \frac{\psi^{\prime}(1+k)}{k^{2}}-\frac{1}{(a-1)} \sum_{k=1}^{\infty} \frac{\psi^{\prime \prime}(1+k)}{k^{2}} .
\end{aligned}
$$

Using the same ideas as the previous lemma together with (1.2), (1.4) and (1.5) we obtain (1.10). To prove (1.11) consider

$$
\begin{gathered}
\sum_{n=1}^{\infty} \frac{H_{n}^{(2)}}{(n+1)^{3}(n+a)^{2}}=\sum_{n=1}^{\infty} \sum_{k=1}^{n} \frac{1}{k^{2}(n+1)^{3}(n+a)^{2}} \\
=\sum_{k=1}^{\infty} \sum_{n=0}^{\infty} \frac{1}{k^{2}(n+1+k)^{3}(n+a+k)^{2}} \\
=\sum_{k=1}^{\infty} \frac{1}{2(a-1)^{4} k^{2}}\left[\begin{array}{c}
6 \psi(a+k)-6 \psi(1+k)-4(a-1) \psi^{\prime}(1+k) \\
-2(a-1) \psi^{\prime}(a+k)-(a-1)^{2} \psi^{\prime \prime}(1+k)
\end{array}\right] \\
=\sum_{k=1}^{\infty} \frac{1}{k^{2}}\left(\begin{array}{c}
\frac{3}{(a-1)^{4}} \sum_{j=1}^{a-1} \frac{1}{(k+j)}+\frac{1}{(a-1)^{3}} \sum_{j=1}^{a-1} \frac{1}{(k+j)^{2}}+\frac{3 \psi^{\prime}(1+k)}{(a-1)^{3}} \\
-\frac{\psi^{\prime \prime}(1+k)}{(a-1)^{2}}
\end{array}\right.
\end{gathered}
$$


and the result (1.11) is attained after utilizing (1.2), (1.4) and (1.5).

Remark 3. Some examples are

$$
\begin{aligned}
& \sum_{n=1}^{\infty} \frac{H_{n}^{(2)}}{n(n+1)^{3}}=\zeta(3)-\frac{3 \zeta(4)}{4}-3 \zeta(2) \zeta(3)+\frac{11}{2} \zeta(5), \\
& \qquad \sum_{n=1}^{\infty} \frac{H_{n}^{(2)}}{(n+1)^{3}(n+5)}=\frac{3}{4} \zeta(2) \zeta(3)-\frac{3 \zeta(4)}{64}-\frac{11}{8} \zeta(5)+\frac{25}{768} \zeta(2)-\frac{2953}{110592} \\
& \sum_{n=1}^{\infty} \frac{H_{n}^{(2)}}{(n+1)^{3}(n+2)^{2}}=\frac{3}{16} \zeta(2) \zeta(3)-\frac{9 \zeta(4)}{256}-\frac{11}{32} \zeta(5)+\frac{635}{9216} \zeta(2)-\frac{9349}{110592} .
\end{aligned}
$$

and

\section{THREE THEOREMS}

We now prove the following three theorems.

Theorem 1. Let $k \in \mathbb{N}, \mathbb{N}:=\{1,2,3, \ldots\}$, then

$$
\begin{aligned}
& \sum_{n=1}^{\infty} \frac{H_{n}^{(2)}}{n\left(\begin{array}{c}
n+k \\
k
\end{array}\right)^{2}} \\
& =\sum_{r=1}^{k} r\left(\begin{array}{c}
k \\
r
\end{array}\right)^{2}\left[\begin{array}{c}
2\left(H_{r-1}-H_{k-r}\right)\left(\zeta(3)+\zeta(2) H_{r-1}+\sum_{j=1}^{r-1} \frac{H_{j}}{j^{2}}\right) \\
-\frac{3}{4} \zeta(4)+\frac{1}{r} \zeta(3)+\zeta(2)\left(\frac{H_{r-1}}{r}+2 H_{r-1}^{(2)}\right) \\
-\sum_{j=1}^{r-1}\left(\frac{H_{j}}{r j^{2}}+\frac{2 H_{j}}{j^{3}}+\frac{H_{j}^{(2)}}{j^{2}}\right)
\end{array}\right]
\end{aligned}
$$

Proof. Consider the following expansion:

$$
\sum_{n=1}^{\infty} \frac{H_{n}^{(2)}}{n\left(\begin{array}{c}
n+k \\
k
\end{array}\right)^{2}}=\sum_{n=1}^{\infty} \frac{(k !)^{2} H_{n}^{(2)}}{n \prod_{r=1}^{k}(n+r)^{2}}=\sum_{n=1}^{\infty} \frac{(k !)^{2} H_{n}^{(2)}}{n\left((n+1)_{k+1}\right)^{2}}
$$

Where $(\alpha)_{r}$ is Pochhammer's symbol given by $(\alpha)_{r}=\alpha(\alpha+1)(\alpha+2) \ldots(\alpha+r-1), r>0,(\alpha)_{0}=1$. Now

$$
\sum_{n=1}^{\infty} \frac{H_{n}^{(2)}}{n\left(\begin{array}{c}
n+k \\
k
\end{array}\right)^{2}}=\sum_{n=1}^{\infty} \frac{(k !)^{2} H_{n}^{(2)}}{n} \sum_{r=1}^{k}\left(\frac{A A_{r}}{(n+r)}+\frac{B B_{r}}{(n+r)^{2}}\right)
$$


where

$$
B B_{r}=\lim _{n \rightarrow(-r)}\left(\frac{(n+r)^{2}}{\prod_{r=1}^{k}(a n+r)^{2}}\right)=\left(\frac{r}{k !}\left(\begin{array}{c}
k \\
r
\end{array}\right)\right)^{2}
$$

and

$$
A A_{r}=\lim _{n \rightarrow(-r)} \frac{d}{d n}\left(\frac{(n+r)^{2}}{\prod_{r=1}^{k}(a n+r)^{2}}\right)=2\left(H_{r-1}-H_{k-r}\right)\left(\frac{r}{k !}\left(\begin{array}{c}
k \\
r
\end{array}\right)\right)^{2} .
$$

Now from (2.2) and using Lemma 1

$$
\begin{aligned}
& \sum_{n=1}^{\infty} \frac{(k !)^{2} H_{n}^{(2)}}{n} \sum_{r=1}^{k}\left(\frac{A A_{r}}{(n+r)}+\frac{B B_{r}}{(n+r)^{2}}\right)=\sum_{r=1}^{k} \sum_{n=1}^{\infty}\left(\begin{array}{c}
\frac{(k !)^{2} H_{n}^{(2)} A A_{r}}{n(n+r)} \\
+\frac{(k !)^{2} H_{n}^{(2)} B B_{r}}{n(n+r)^{2}}
\end{array}\right) \\
& =\sum_{r=1}^{k} \sum_{n=1}^{\infty}\left(\frac{2\left(H_{r-1}-H_{k-r}\right)\left(r\left(\begin{array}{c}
k \\
r
\end{array}\right)\right)^{2} H_{n}^{(2)}}{n(n+r)}+\frac{\left(r\left(\begin{array}{c}
k \\
r
\end{array}\right)\right)^{2} H_{n}^{(2)}}{n(n+r)^{2}}\right)
\end{aligned}
$$

and using (1.6) and (1.7) we obtain the result.

Remark 4. For the case $k=5$, we have the result

$$
\sum_{n=1}^{\infty} \frac{H_{n}^{(2)}}{n\left(\begin{array}{c}
n+5 \\
5
\end{array}\right)^{2}}=\zeta(3)-\frac{945}{2} \zeta(4)+1750 \zeta(2)-\frac{16370447}{6912}
$$

Now we consider the following Theorem 1.

Theorem 2. Let $k \in \mathbb{N} \backslash\{1\}, \mathbb{N}:=\{1,2,3, \ldots\}$, then

$$
\sum_{n=1}^{\infty} \frac{H_{n}^{(2)}}{\left(\begin{array}{c}
n+k \\
k
\end{array}\right)^{2}}
$$




$$
=\sum_{r=2}^{k}\left(r\left(\begin{array}{c}
k \\
r
\end{array}\right)\right)^{2}\left[\begin{array}{c}
2\left(H_{r-2}-H_{k-r}\right)\left(\begin{array}{c}
\frac{3(r-1) \zeta(4)}{4}-\zeta(2) H_{r-1} \\
+\sum_{j=1}^{r-1} \frac{H_{j}}{j^{2}}
\end{array}\right) \\
\frac{3 \zeta(4)}{2}-\frac{2 \zeta(2) H_{r-1}}{(r-1)}-2 \zeta(2) H_{r-1}^{(2)} \\
+\sum_{j=1}^{r-1}\left(\frac{2 H_{j}}{(r-1) j^{2}}+\frac{2 H_{j}}{j^{3}}+\frac{H_{j}^{(2)}}{j^{2}}\right)
\end{array}\right]
$$

Proof. Consider the following expansion:

$$
\sum_{n=1}^{\infty} \frac{H_{n}^{(2)}}{\left(\begin{array}{c}
n+k \\
k
\end{array}\right)^{2}}=\sum_{n=1}^{\infty} \frac{(k !)^{2} H_{n}^{(2)}}{(n+1)^{2} \prod_{r=2}^{k}(n+r)^{2}}=\sum_{n=1}^{\infty} \frac{(k !)^{2} H_{n}^{(2)}}{(n+1)^{2}\left((n+2)_{k+1}\right)^{2}}
$$

Now

$$
\sum_{n=1}^{\infty} \frac{H_{n}^{(2)}}{\left(\begin{array}{c}
n+k \\
k
\end{array}\right)^{2}}=\sum_{n=1}^{\infty} \frac{(k !)^{2} H_{n}^{(2)}}{(n+1)^{2}} \sum_{r=2}^{k}\left(\frac{A_{r}}{(n+r)}+\frac{B_{r}}{(n+r)^{2}}\right)
$$

where

$$
B_{r}=\lim _{n \rightarrow(-r)}\left(\frac{(n+r)^{2}}{\prod_{r=2}^{k}(a n+r)^{2}}\right)=\left(\frac{2}{k !}\left(\begin{array}{l}
k \\
r
\end{array}\right)\left(\begin{array}{l}
r \\
2
\end{array}\right)\right)^{2}
$$

and

$$
A_{r}=\lim _{n \rightarrow(-r)} \frac{d}{d n}\left(\frac{(n+r)^{2}}{\prod_{r=2}^{k}(a n+r)^{2}}\right)=2\left(H_{r-2}-H_{k-r}\right)\left(\frac{2}{k !}\left(\begin{array}{c}
k \\
r
\end{array}\right)\left(\begin{array}{c}
r \\
2
\end{array}\right)\right)^{2}
$$

Now from (2.4) and using Lemma 2 


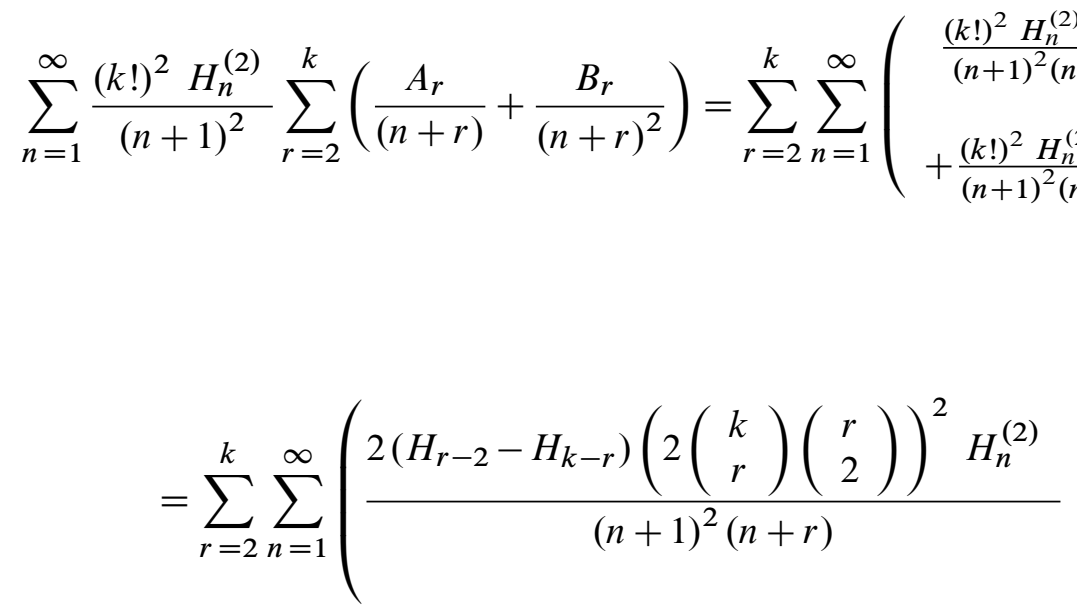

$$
\begin{aligned}
& \left.+\frac{\left(2\left(\begin{array}{l}
k \\
r
\end{array}\right)\left(\begin{array}{l}
r \\
2
\end{array}\right)\right)^{2} H_{n}^{(2)}}{(n+1)^{2}(n+r)^{2}}\right)
\end{aligned}
$$

and using (1.8) and (1.9) we obtain the result.

Remark 5. An example for Theorem 2 is

$$
\sum_{n=1}^{\infty} \frac{H_{n}^{(2)}}{\left(\begin{array}{c}
n+5 \\
5
\end{array}\right)^{2}}=\frac{2625}{2} \zeta(4)-\frac{179375}{36} \zeta(2)+\frac{140498725}{20736}
$$

The final theorem follows.

Theorem 3. Let $k \in \mathbb{N} \backslash\{1\}, \mathbb{N}:=\{1,2,3, \ldots\}$, then 


$$
\left.\begin{array}{c}
\sum_{n=1}^{\infty} \frac{H_{n}^{(2)}}{(n+1)\left(\begin{array}{c}
n+k \\
k
\end{array}\right)^{2}} \\
=\sum_{r=2}^{k}\left(r\left(\begin{array}{c}
k \\
r
\end{array}\right)\right)^{2} \\
2\left(\begin{array}{c}
3(r-1) \zeta(2) \zeta(3)-\frac{11(r-1) \zeta(5)}{2}-\frac{3 \zeta(4)}{4} \\
+\frac{\zeta(2) H_{r-1}}{(r-1)}-\frac{1}{(r-1)} \sum_{j=1}^{r-1} \frac{H_{j}}{j^{2}}
\end{array}\right) \\
3 \zeta(2) \zeta(3)-\frac{11 \zeta(5)}{2}-\frac{9 \zeta(4)}{4(r-1)}+\frac{2 \zeta(2) H_{r-1}^{(2)}}{(r-1)} \\
+\frac{3 \zeta(2) H_{r-1}}{(r-1)^{2}}-\frac{1}{(r-1)} \sum_{j=1}^{r-1}\left(\frac{3 H_{j}}{(r-1) j^{2}}+\frac{2 H_{j}}{j^{3}}+\frac{H_{j}^{(2)}}{j^{2}}\right)
\end{array}\right]
$$

Proof. Consider the following expansion:

$$
\begin{aligned}
& \sum_{n=1}^{\infty} \frac{H_{n}^{(2)}}{(n+1)\left(\begin{array}{c}
n+k \\
k
\end{array}\right)^{2}}=\sum_{n=1}^{\infty} \frac{(k !)^{2} H_{n}^{(2)}}{(n+1)^{3} \prod_{r=2}^{k}(n+r)^{2}} \\
& =\sum_{n=1}^{\infty} \frac{(k !)^{2} H_{n}^{(2)}}{(n+1)^{3}\left((n+2)_{k+1}\right)^{2}} .
\end{aligned}
$$

Now

$$
\sum_{n=1}^{\infty} \frac{H_{n}^{(2)}}{(n+1)\left(\begin{array}{c}
n+k \\
k
\end{array}\right)^{2}}=\sum_{n=1}^{\infty} \frac{(k !)^{2} H_{n}^{(2)}}{(n+1)^{3}} \sum_{r=2}^{k}\left(\frac{A_{r}}{(n+r)}+\frac{B_{r}}{(n+r)^{2}}\right)
$$

where $B_{r}$ and $A_{r}$ are given respectively by (2.5) and (2.6). Now from (2.8) and using Lemma 3

$$
\sum_{n=1}^{\infty} \frac{(k !)^{2} H_{n}^{(2)}}{(n+1)^{3}} \sum_{r=2}^{k}\left(\frac{A_{r}}{(n+r)}+\frac{B_{r}}{(n+r)^{2}}\right)=\sum_{r=2}^{k} \sum_{n=1}^{\infty}\left(\begin{array}{c}
\frac{(k !)^{2} H_{n}^{(2)} A_{r}}{(n+1)^{3}(n+r)} \\
+\frac{(k !)^{2} H_{n}^{(2)} B_{r}}{(n+1)^{3}(n+r)^{2}}
\end{array}\right)
$$

and using (1.10) and (1.11) we obtain the result (2.7). 
Remark 6. An example for Theorem 3 is

$$
\begin{aligned}
& \sum_{n=1}^{\infty} \frac{H_{n}^{(2)}}{(n+1)\left(\begin{array}{c}
n+5 \\
5
\end{array}\right)^{2}} \\
& =75 \zeta(2) \zeta(3)-\frac{275}{2} \zeta(5)-\frac{13125}{16} \zeta(4)+\frac{5050925}{1728} \zeta(2)-\frac{40704625}{10368} .
\end{aligned}
$$

\section{REFERENCES}

[1] H. Alzer, D. Karayannakis, and H. M. Srivastava, "Series representations for some mathematical constants," J. Math. Anal. Appl., vol. 320, no. 1, pp. 145-162, 2006.

[2] H. Alzer and S. Koumandos, "Series and product representations for some mathematical constants," Period. Math. Hung., vol. 58, no. 1, pp. 71-82, 2009.

[3] D. H. Bailey, J. M. Borwein, and R. Girgensohn, "Experimental evaluation of Euler sums," Exp. Math., vol. 3, no. 1, pp. 17-30, 1994.

[4] D. Borwein and J. M. Borwein, "On an intriguing integral and some series related to $\zeta(4)$," Proc. Am. Math. Soc., vol. 123, no. 4, pp. 1191-1198, 1995.

[5] J. Choi, "Certain summation formulas involving harmonic numbers and generalized harmonic numbers," Appl. Math. Comput., vol. 218, no. 3, pp. 734-740, 2011.

[6] J. Choi and H. M. Srivastava, "Explicit evaluation of Euler and related sums," Ramanujan J., vol. 10, no. 1, pp. 51-70, 2005.

[7] W. Chu and Q. Yan, "Combinatorial identities on binomial coefficients and harmonic numbers," Util. Math., vol. 75, pp. 51-66, 2008.

[8] L. Euler, Opera omnia. Series prima: Opera mathematica. Vol. XV: Commentationes analyticae ad theoriam seriarum infinitarum pertinentes. Berlin: B. G. Teubner, 1911.

[9] P. Flajolet and B. Salvy, "Euler sums and contour integral representations," Exp. Math., vol. 7, no. 1, pp. 15-35, 1998.

[10] T.-X. He, L. C. Hsu, and D. Yin, "A pair of operator summation formulas and their applications," Comput. Math. Appl., vol. 58, no. 7, pp. 1340-1348, 2009.

[11] M. J. Kronenburg, "Some combinatorial identities some of which involving harmonic numbers," 2011. [Online]. Available: http://arXiv.1103.1268V1

[12] T. Mansour, "Identities on harmonic and $q$-harmonic number sums," Afr. Mat., 2011.

[13] E. Munarini, "Riordan matrices and sums of harmonic numbers," Appl. Anal. Discrete Math., vol. 5, pp. 176-200, 2011.

[14] A. Sofo, Computational techniques for the summation of series. New York: Kluwer Academic/Plenum Publishers, 2003.

[15] A. Sofo, "Harmonic sums and integral representations," J. Appl. Analysis, vol. 16, pp. 265-277, 2010.

[16] A. Sofo, "Summation formula involving harmonic numbers," Anal. Math., vol. 37, no. 1, pp. 5164, 2011.

[17] J. Sondow and E. W. Weisstein, "Harmonic number. From MathWorld-A Wolfram Web Rescources." [Online]. Available: http://mathworld.wolfram.com/HarmonicNumber.html

[18] W. Wang, "Riordan arrays and harmonic number identities," Comput. Math. Appl., vol. 60, no. 5, pp. 1494-1509, 2010.

[19] W. E. Weisstein, "Euler Sum. From MathWorld-A Wolfram Web Resource.” [Online]. Available: http://mathworld.wolfram.com/EulerSum.html 
[20] D.-Y. Zheng, "Further summation formulae related to generalized harmonic numbers," J. Math. Anal. Appl., vol. 335, no. 1, pp. 692-706, 2007.

\section{Author's address}

Anthony Sofo

Victoria University College., Victoria University, PO Box 14428, Melbourne City,, VIC 8001, Australia.

E-mail address: anthony. sofo@vu.edu.au 